\title{
Liver Failure and Conjugated Hyperbilirubinemia in a Preterm Neonate: Role of Early IVIG and Exchange Transfusion
}

\author{
Jayasree Nair, MD ${ }^{1}$ Vasantha H.S. Kumar, MD ${ }^{1}$ \\ ${ }^{1}$ Department of Pediatrics, John R. Oishei Children's Hospital, \\ Buffalo, New York \\ Am J Perinatol Rep 2018;8:e95-e98.
}

\begin{abstract}
Address for correspondence Jayasree Nair, MD, Division of Neonatology, Department of Pediatrics, John R. Oishei Children's Hospital of Buffalo, University at Buffalo, Conventus Building 1001 Main Street Buffalo, NY 14203 (e-mail: jnair@upa.chob.edu).
\end{abstract}

\begin{abstract}
Neonatal liver failure (NLF) is a rare diagnosis but carries with it significant risks of mortality and morbidity. Common etiologies for NLF include metabolic causes, gestational alloimmune liver disease (GALD or neonatal hemochromatosis), and viral infections. We report a case of liver failure in a premature infant with abnormal iron profile within 48 hours of birth. Lack of accepted guidelines for the initial management of severe jaundice with a high direct component in the first week after birth made treatment challenging. The infant underwent intensive phototherapy along with four exchange transfusions (ET) and two courses of intravenous immunoglobulins (IVIG).

\section{Keywords}

- hyperbilirubinemia

- hemochromatosis

- exchange transfusion

- IVIG

- GALD

- neonatal

- liver failure

The clinical goals were to keep total bilirubin values $\leq 20 \mathrm{mg} / \mathrm{dL}$ in this premature neonate and to minimize the risk of bilirubin-induced neurologic dysfunction and decompensated liver failure. Abnormal iron studies and later magnetic resonance imaging were suggestive of GALD. Liver functions improved over time with normal neurodevelopmental assessment at 3 years of age. To conclude, in infants with NLF soon after birth, earlier consideration of IVIG/ET in the first few days may be beneficial. Larger multicenter data analyses are required to formulate treatment guidelines and indications for phototherapy, ET, and IVIG in sick neonates with NLF.
\end{abstract}

Neonatal liver failure (NLF) or neonatal acute liver failure is diagnosed in infants with loss of vital liver functions and coagulopathy. Presence of hepatic cirrhosis and lack of encephalopathy do not preclude a diagnosis of NLF. This condition is a common indication for liver transplantation. ${ }^{1}$ Gestational alloimmune liver disease (GALD) presenting as neonatal hemochromatosis $(\mathrm{NH})$ is a major cause of NLF. This clinically distinct disease of unclear etiology is characterized by NLF with extrahepatic siderosis. ${ }^{1}$ The disease is thought to be an alloimmune liver injury in the fetus mediated by immunoglobulin $\mathrm{G}$ (IgG) to the hepatocyte antigen. ${ }^{2}$ Recently, exchange transfusion (ET) and intravenous immunoglobulin (IVIG) have been shown to rescue these infants from liver transplantation with improved prognosis. ${ }^{3}$ Our case report highlights the dilemma the clinician faces in treating severe hyperbilirubi-

received

August 31, 2017 accepted after revision March 4, 2018 nemia with a high direct component in the absence of established guidelines for phototherapy, ET, and IVIG.

\section{Case}

A female baby was born at 32 2/7 weeks' gestation to a 35-yearold G3Ab2 Caucasian mother with premature onset of labor by cesarean section for nonreassuring fetal heart rate. Her prenatal screening laboratory tests were negative; she was noted to be a chronic cigarette smoker with no history of alcohol use. She was on methadone during pregnancy with a urine toxicology screen positive for cocaine a month prior to delivery. Maternal history was significant for hypothyroidism, depression, hypertension, anemia, gastric ulcer, endometriosis, cholecystectomy, and a motor vehicle accident. She had a history
Copyright $\odot 2018$ by Thieme Medical Publishers, Inc., 333 Seventh Avenue, New York, NY 10001, USA. Tel: +1(212) 584-4662.
License terms

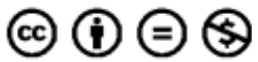

10.1055/s-0038-1649339. ISSN 2157-6998. 
of pancreatitis and "liver disease" for which she was on ursodiol. A liver biopsy, done 2 years prior to delivery, revealed periportal fibrosis. Her liver function tests (LFTs) 2 months prior to delivery (PTD) were within normal range. She was on ursodiol, prenatal vitamins, methadone, prozac, pregabalin, gabapentin, lansoprazole, and doxepin during pregnancy.

The baby was intubated and suctioned for meconium. The cord gas was 7.03/-13 with Apgar scores of $4,{ }^{1} 5,,^{5} 8,^{10}$ respectively. Initial physical exam was significant for facial bruising, craniotabes, hepatomegaly, splenomegaly (liver $3 \mathrm{~cm}$, spleen $2 \mathrm{~cm}$ below the costal margin), ascites, and edematous extremities. Infant's birth weight (1415 g-19th centile) and length ( $42 \mathrm{~cm}-34$ th centile) were appropriate for gestational age ${ }^{4}$ with microcephaly (head circumference: $30 \mathrm{~cm}$, 2nd centile). Infant was initially placed on conventional ventilator, but rapidly worsened with respiratory failure and required high-frequency ventilation. The chest X-ray was suggestive of respiratory distress syndrome and surfactant was administered prior to transport. A mixed metabolic and respiratory acidosis was noted (ABG: 6.97/64/68/-18.2) which improved after saline infusion and bicarbonate bolus to $7.29 /$ 48/27/-3.7. Upon transfer to level-4 neonatal intensive care unit, the laboratory test results demonstrated thrombocytopenia, low hematocrit, disseminated intravascular coagulation
(DIC) ( - Table 1), and abnormal liver function tests ( - Table $\mathbf{2}$ ). No hypoglycemia was noted on admission to the intensive care unit (blood glucose-84 mg/dL).

\section{Hyperbilirubinemia}

Infant was jaundiced with small, well-defined purplish, flat rash on the face and extremities (blueberry muffin-like) with decreased muscle tone. The initial bilirubin was $26.8 \mathrm{mg} / \mathrm{dL}$ with a direct component of $16.7 \mathrm{mg} / \mathrm{dL}$. Phototherapy was initiated in preparation for double-volume exchange transfusion (DVET) in this infant. The progression of total bilirubin in the first week after birth is depicted in - Fig. 1A. Infant underwent a total of four DVETs at $\sim 24,30,84$, and 96 hours of age. The pre-exchange transfusion total bilirubin levels were $23.2 \mathrm{mg} / \mathrm{dL}$ (24 h), 20.4 (30 h), 20.1/13.3 (84 h), and 20.3/12.0 (96h) respectively ( $\mathbf{F i g . 1 A}$ ). Infant received two doses of IVIG 0.5 grams $/ \mathrm{kg}$ on day 2 (bilirubin-18 $\mathrm{mg} / \mathrm{dL}$ ) and on day 4 (bilirubin-18 mg/dL) after birth. -Fig. 1B illustrates the progression of total and direct bilirubin during the infant's stay in the hospital. Diagnostic evaluation to rule out etiologies leading to NLF was initiated ( - Table 1). Mild hemolysis was evidenced by initial high reticulocyte count and nucleated red blood cells on the peripheral smear. A high serum ferritin level,

Table 1 Summary of investigations in infant with liver failure and hyperbilirubinemia

\begin{tabular}{|c|c|}
\hline Investigation & Values \\
\hline $\mathrm{Hb} /$ Hematocrit & $10.9 \mathrm{~g} / \mathrm{dL} / 31.1 \%$ (nL:15-24/44-70\%) \\
\hline WBC count & $\begin{array}{l}14 \times 10^{9} / \mathrm{L} \text { (nL: } 9.1-34.3 \text { ); segs-41\%; bands-14\%; lymphs-32\%; monos-12\%; } \\
\text { metamyelo-1\%; NRbc-132 }\end{array}$ \\
\hline Platelet count & $50 \times 10^{9} / \mathrm{L}(\mathrm{nL}: 150-450)$ \\
\hline $\begin{array}{l}\text { Reticulocyte count/absolute } \\
\text { reticulocyte count }\end{array}$ & $\begin{array}{l}\mathrm{D} 2-7.5 \% / 221 \times 10^{9} / \mathrm{L}(\mathrm{nL}: 60-190) \\
\mathrm{D} 3-1.3 \% / 58 \times 10^{9} / \mathrm{L}\end{array}$ \\
\hline Peripheral smear & Slight aniso, micro, and poikilocytosis \\
\hline Clotting studies on day 1 & $\begin{array}{l}\text { PT-34 second (nL: } 11-15) \text {; APTT-98 second (nL: 25-34); } \\
\text { fibrinogen- } 80 \mathrm{mg} / \mathrm{dL}(\mathrm{nL}: 200-470)\end{array}$ \\
\hline Factor assays on day 7 & $\begin{array}{l}\text { Factor II activity-39\% (nL:75-135); Factor IX activity-22\% (nL: 60-160); } \\
\text { Factor VII activity-33\% (nL: 70-170); Factor X activity-36\% (70-140) }\end{array}$ \\
\hline Ferritin & D2: 2652 (nL: 25-200) ng/mL \\
\hline Iron studies & $\begin{array}{l}\text { D5-Serum Fe-197 (nL: } 20-140) \mu \mathrm{g} / \mathrm{dL} ; \mathrm{TIBC}-243(\mathrm{~nL}: 250-450) \mu \mathrm{g} / \mathrm{dL} ; \% \mathrm{TS}-81 \% \\
\text { (nL: } 21-55 \%) ; 3 \text { years-serum Fe-116 } \mu / \mathrm{dL}(20-140) ; \mathrm{TIBC}-335 \mu \mathrm{g} / \mathrm{dL}(250-450) \text {; } \\
\% \text { TS-35\% (nL: 21-55) }\end{array}$ \\
\hline Infectious screen & RPR-NR; Treponema pallidum antibody IgG-NR; Treponema gondi IgM-Negative; \\
\hline Viral studies & $\begin{array}{l}\text { Epstein-Barr IgM IFA-neg; coxsackievirus group B1/B2/B3/B4/B5/B6-neg; } \\
\text { Hepatitis A/B/C-neg; parvovirus B19-neg; herpes simplex virus type 1/2-neg }\end{array}$ \\
\hline$\alpha-1$ Fetoprotein & 2049 ng/mL (nL: 0-15) \\
\hline Blood group & O positive; DCT-neg; Ab. Screen-neg \\
\hline Others & $\begin{array}{l}\text { Transferrin-154 mg/dL (120-250); } \alpha \text {-1-antitrypsin-90 mg/dL (nL: } 90-120) ; \\
\text { serum ammonia-127 } \mu \mathrm{g} / \mathrm{L}(10-155) ; \text { lactate-1.3 mmol/L (nL: } 0.5-2) ; \\
\text { pyruvate-1.4 mg/dL (nL: 0.3-0.7); CRP-6.66 mg/L (nL: <10); } \\
\text { tyrosine-497 (nL:40-125) } \mu \text { mol /L; phenylalanine-4.15 (nL: } 2-6) \mathrm{mg} / \mathrm{dL} ; \\
\text { newborn screen-normal; galactose-1-phosphate uridyltransferase activity-normal }\end{array}$ \\
\hline Cultures (blood/urine/stools) & Negative; CMV viral culture-neg \\
\hline
\end{tabular}

Abbreviations: CRP, C-reactive protein; DCT, direct Coombs test; IgG, immunoglobulin G; INR, international normalized ratio; $\mathrm{nL}$, normal; NR, nonreactive; PT, prothrombin time; PTT, partial thromboplastin time; TIBC, total iron binding capacity; TS, transferrin saturation. 
Table 2 Progression of liver function tests and clotting studies in infant with neonatal liver failure

\begin{tabular}{|l|l|l|l|l|l|l|l|l|l|l|}
\hline Age & $\begin{array}{l}\text { Alk } \\
\mu / \mathrm{L}\end{array}$ & $\begin{array}{l}\text { AST } \\
\mu / \mathrm{L}\end{array}$ & $\begin{array}{l}\text { ALT } \\
\mu / \mathrm{L}\end{array}$ & $\begin{array}{l}\text { GGT } \\
\mu / \mathrm{L}\end{array}$ & $\begin{array}{l}\text { TP } \\
\mathbf{g} / \mathrm{dL}\end{array}$ & $\begin{array}{l}\text { Alb } \\
\mathbf{g} / \mathbf{d L}\end{array}$ & $\begin{array}{l}\text { PT } \\
\text { Sec }\end{array}$ & $\begin{array}{l}\text { PTT } \\
\text { Sec }\end{array}$ & $\begin{array}{l}\text { INR } \\
\mathbf{m g} / \mathbf{d L}\end{array}$ \\
\hline Day1 & 269 & 1418 & 206 & 30 & 4 & 1.8 & 18.9 & 40.5 & 1.7 & 164 \\
\hline Day3 & 160 & 101 & 42 & 4.6 & 2.0 & & 17.5 & 41.2 & 1.53 & 273 \\
\hline Day 4 & 224 & 135 & 44 & 18 & 5.2 & 2.5 & 19.2 & 42.3 & 1.73 & 208 \\
\hline Day 11 & 737 & 184 & 87 & 40 & 5.8 & 2.4 & 17.6 & 40.2 & 1.54 & 205 \\
\hline Day 18 & 625 & 199 & 94 & 47 & 6.1 & 3.0 & 13.3 & 32.9 & 1.06 & 241 \\
\hline Day 25 & 482 & 451 & 170 & 80 & 5.5 & 2.9 & 12.1 & 32.6 & 0.94 & 260 \\
\hline Day 32 & 475 & 229 & 150 & 102 & 5.4 & 3.3 & 12.0 & 31.0 & 0.93 & 255 \\
\hline Day 37 & 409 & 127 & 89 & 177 & 5.5 & 3.4 & & & & \\
\hline 7 months & 249 & 40 & 24 & 9 & 6.2 & 4.3 & 13.1 & 31.8 & 1.04 & \\
\hline 3 years & 289 & 36 & 26 & & 7.0 & 4.5 & & & & \\
\hline
\end{tabular}

Abbreviations: Alb, albumin; Alk, alkaline phosphatase; ALT, alanine aminotransferase; AST, aspartate aminotransferase; Fib, fibrinogen; GGT, gamma-glutamyl transpeptidase; INR, international normalized ratio; PT, prothrombin time; PTT, partial thromboplastin time; TP, total proteins.
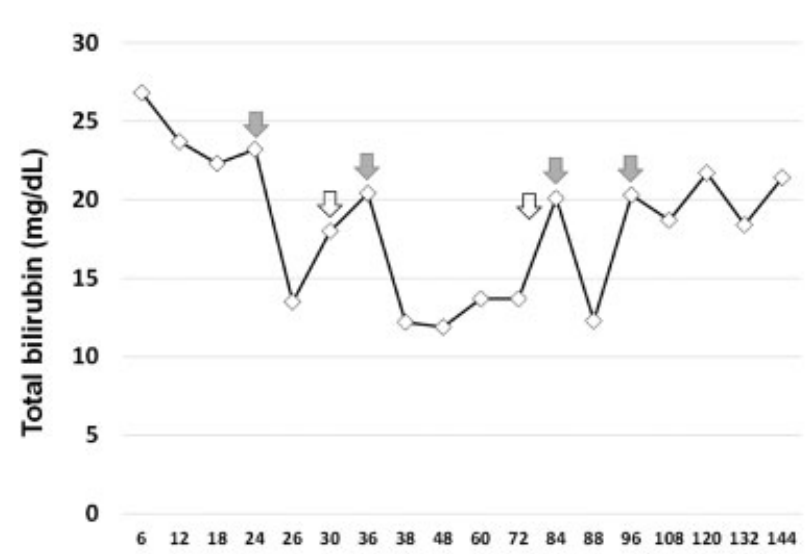

A Time in hours after birth
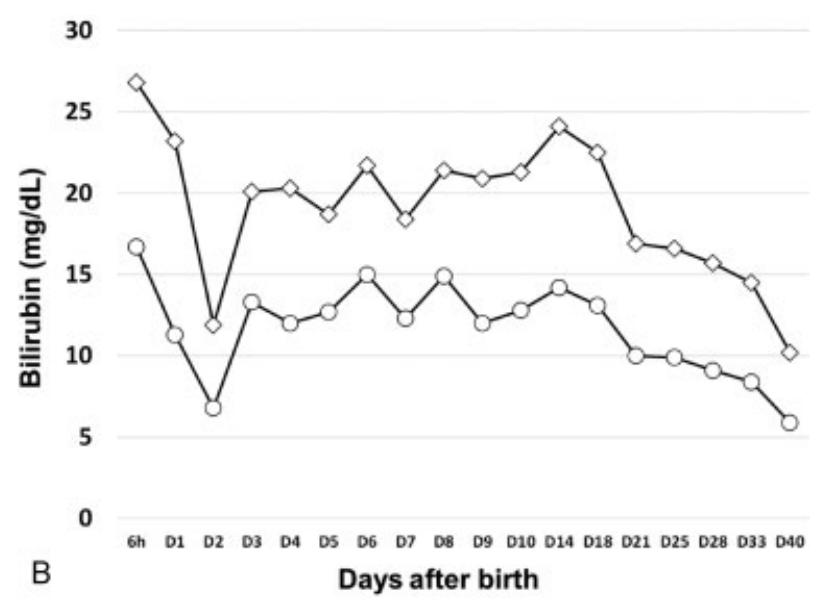

Fig. 1 The progression of total bilirubin (open diamond) in the first 7 days of life (A) along with direct bilirubin (open circles) (B) in the first month of life after birth. We attempted to keep total bilirubin $\leq 20$ $\mathrm{mg} / \mathrm{dL}$ in the first week after birth. Two courses of immunoglobulin were administered (open arrows) (A) and four double-volume exchange transfusions (shaded arrows) performed on the infant. Following each exchange transfusions, total bilirubin rebounded higher to peak at 2 weeks and eventually decreased over time (B). high serum iron, low total iron binding capacity, and high transferrin saturation were noted with repeated abnormal coagulation screens. DIC persisted for 2 weeks with infant receiving multiple fresh frozen plasma and platelet transfusions prior to normalization of coagulation profile. Abdominal ultrasound demonstrated enlarged liver with no focal masses and no dilatation of intrahepatic biliary ducts. The spleen was enlarged for age and the rest of the organs were normal. Ventricles were normal on head ultrasound with no hemorrhage. Serial echocardiograms revealed normal cardiac anatomy and function. Magnetic resonance imaging of the abdomen demonstrated signal intensity of the liver less than that of the skeletal muscle, consistent with hemochromatosis of the liver. A mucosal biopsy was planned but had to be deferred due to poor yield and improving liver function, after consultation with the transplant center.

\section{Convalescent Care}

DIC resolved and LFTs improved over time (-Table 2 ). Infant was on a high calorie enteral diet $(30 \mathrm{Kcal} / \mathrm{oz})$ for failure to thrive at discharge, later requiring a percutaneous gastrostomy tube at 7 months of age. She was found to have bilateral hearing impairment at mid to high frequencies on BAER follow-up. Infant was followed in the neurodevelopmental clinic at our institute on a regular basis after discharge. At her 29-month assessment, using CAT/CLAMS (clinical adaptive test/clinical linguistic and auditory milestone scale), she had a developmental quotient of 90 (normal $\geq 85$ ).

\section{Discussion}

Though a definitive diagnosis could not be established in this neonate with NLF, intrauterine growth restriction, prematurity, and hyperferritinemia with abnormal iron status may be suggestive of GALD-NH. Hepatic siderosis, as seen in this infant, can be present in many other causes of NLF. Evidence 
of extrahepatic siderosis, such as in the exocrine pancreas, minor salivary glands, and the respiratory tree besides the liver, ${ }^{5}$ could not be documented. Notably, the infant had elevated tyrosine levels and persistent patency of ductus venosus both seen in GALD-NH. ${ }^{1}$ GALD is thought to be an alloimmune liver injury in the fetus, mediated by maternal IgG antibodies directed against the fetal hepatocytes. ${ }^{2}$ The antigen appears to be a hepatocyte-specific protein uniquely expressed by the fetal hepatocytes; once sensitized to fetal antigen, reactive IgG is transferred to the fetus activating the complement pathway, resulting in hepatocyte injury, a defining feature of GALD. GALD has emerged as one of the commonest etiologies of NLF (60-90\%). ${ }^{6}$ However, nonGALD etiology of NLF should be considered in the diagnosis and systematic workup of these infants. The prognosis for untreated NLF is generally poor with many infants requiring liver transplantation. In our infant, due to additional complications of prematurity, growth restriction and multiorgan failure, liver transplantation was not an immediate consideration. A therapeutic regimen of ET followed by IVIG has been introduced to eliminate suspected alloantibodies inducing GALD-NH and has been shown to reduce the need for liver transplantation. ${ }^{3}$ Administration of IVIG followed by ET has been described on day 25 after birth in an infant with $\mathrm{NH}$, in addition to chelation-antioxidant therapy from day $10{ }^{7}$ The combination of ET and IVIG, as in our infant, may help in physical removal of alloantibodies from the neonatal circulation and have an immunomodulatory effect.

In the largest case series published to date, ET/IVIG appears to improve outcome and reduce the need for liver transplantation. ${ }^{3}$ In the same case series, ET/IVIG was administered at a mean of $15 \pm 9$ days and only six infants received DVET. ${ }^{3}$ The case that we have presented is unique in several aspects. The infant received multiple DVETs/IVIG all in the first week of life based on American Academy of Pediatrics (AAP)-recommended bilirubin guidelines. ${ }^{8}$ The AAP recommends not subtracting direct bilirubin from the total bilirubin measurements. However, if the direct bilirubin is $>50 \%$, no good data exist to provide guidance. ${ }^{8}$ Hence, we decided to proceed with ET/IVIG in this case with a strong suspicion for GALD.

Infants with NLF may be extremely sick with total bilirubin values frequently exceeding $25 \mathrm{mg} / \mathrm{dL}$. Though there is a high direct component, the treatment protocol for these infants follows AAP hyperbilirubinemia guidelines based on total bilirubin values. ${ }^{8}$ Condition-specific threshold values for bilirubin-induced neurologic dysfunction (BIND) have not been identified and optimal operational thresholds for treatment are yet to be delineated. ${ }^{9}$ In population-based studies, the risk of chronic bilirubin encephalopathy or kernicterus varied from 1 in 17.6 in Canada ${ }^{9,10}$ to 1 in 16.2 in Denmark ${ }^{9,11}$ for a total serum bilirubin level $>25 \mathrm{mg} / \mathrm{dL}$. In suspected GALD, ongoing liver injury from maternal alloantibodies may contribute to rising bilirubin, leading to rebound hyperbilirubinemia after ET necessitating repeated ETs. Due to high risk of BIND in immune jaundice, intensive phototherapy was continued in this infant for the first 2 weeks, in spite of a predominantly direct component. IVIG infusions were administered 2 to $3 \mathrm{mg} / \mathrm{dL}$ below the ET values of around $20 \mathrm{mg} / \mathrm{dL}$. A more rapid normalization of clotting studies was noted following multiple DVETs (- Table 2), suggesting an immune etiology. The infant had essentially normal liver function by 6 months of age. We propose that earlier administration of IVIG and ET performed in the first week of life not only reduces the morbidity from liver injury and multiorgan failure but also helps in preservation and faster recovery of liver function over time. In this infant, we strived to keep total bilirubin values $\leq 20 \mathrm{mg} / \mathrm{dL}$, as the goal to decrease potential for BIND. The infant had bilateral hearing impairment found typically in infants with hyperbilirubinemia; however, her neurodevelopmental assessment at 29 months was reassuring.

To conclude, NLF is a potentially life-threatening condition with a few established treatment guidelines. GALD-NH should be suspected in neonates presenting in liver failure at birth. Along with supportive therapy for NLF, earlier ET and IVIG may help to improve prognosis, decrease incidence of BIND and need for liver transplantation in these infants. Due to the rarity of this diagnosis, pooling of data and large multicenter analyses are required to formulate management protocols for NLF.

\section{Source of Funding}

None.

Conflicts of Interests

None.

\section{References}

1 Whitington PF. Neonatal hemochromatosis: a congenital alloimmune hepatitis. Semin Liver Dis 2007;27(03):243-250

2 Feldman AG, Whitington PF. Neonatal hemochromatosis. J Clin Exp Hepatol 2013;3(04):313-320

3 Rand EB, Karpen SJ, Kelly S, et al. Treatment of neonatal hemochromatosis with exchange transfusion and intravenous immunoglobulin. J Pediatr 2009;155(04):566-571

4 Fenton TR. A new growth chart for preterm babies: Babson and Benda's chart updated with recent data and a new format. BMC Pediatr 2003;3:13

5 Goldfischer S, Grotsky HW, Chang $\mathrm{CH}$, et al. Idiopathic neonatal iron storage involving the liver, pancreas, heart, and endocrine and exocrine glands. Hepatology 1981;1(01):58-64

6 Taylor SA, Whitington PF. Neonatal acute liver failure. Liver Transpl 2016;22(05):677-685

7 Babor F, Hadzik B, Stannigel H, Mayatepek E, Hoehn T. Successful management of neonatal hemochromatosis by exchange transfusion and immunoglobulin: a case report. J Perinatol 2013;33(01):83-85

8 American Academy of Pediatrics Subcommittee on Hyperbilirubinemia. Management of hyperbilirubinemia in the newborn infant 35 or more weeks of gestation. Pediatrics 2004;114(01):297-316

9 Bhutani VK, Johnson L. Kernicterus in the 21st century: frequently asked questions. J Perinatol 2009;29(Suppl 1):S20-S24

10 Sgro M, Campbell D, Shah V. Incidence and causes of severe neonatal hyperbilirubinemia in Canada. CMAJ 2006;175(06):587-590

11 Ebbesen $F$. Recurrence of kernicterus in term and near-term infants in Denmark. Acta Paediatr 2000;89(10):1213-1217 